\title{
An Approximation of Hedberg's Type in Sobolev Spaces with Variable Exponent and Application
}

\author{
Abdelmoujib Benkirane, Mostafa El Moumni, and Aziz Fri \\ Department of Mathematics, Laboratory LAMA, Faculty of Sciences Dhar El Mahraz, University Sidi Mohamed Ben Abdellah, \\ BP 1796, 30000 Atlas Fez, Morocco \\ Correspondence should be addressed to Aziz Fri; friazizon@gmail.com
}

Received 20 February 2014; Accepted 4 April 2014; Published 30 April 2014

Academic Editor: Juntao Sun

Copyright (C) 2014 Abdelmoujib Benkirane et al. This is an open access article distributed under the Creative Commons Attribution License, which permits unrestricted use, distribution, and reproduction in any medium, provided the original work is properly cited.

The aim of this paper is to extend the usual framework of PDE with $A(u)=-\operatorname{div} a(x, u, \nabla u)$ to include a large class of cases with $A(u)=\sum_{|\beta| \leq \alpha}(-1)^{|\beta|} D^{\beta} A_{\beta}\left(x, u, \nabla u, \ldots, \nabla^{\alpha} u\right)$, whose coefficient $A_{\beta}$ satisfies conditions (including growth conditions) which guarantee the solvability of the problem $A(u)=f$. This new framework is conceptually more involved than the classical one includes many more fundamental examples. Thus our main result can be applied to various types of PDEs such as reaction-diffusion equations, Burgers type equation, Navier-Stokes equation, and $p$-Laplace equation.

\section{Introduction}

This paper is motivated by the study of the unilateral problem associated with the following equation:

$$
A(u)+g(x, u)=f .
$$

We show the existence of variational solutions of this elliptic boundary value problem for strongly elliptic systems of order $2 m$ on a domain $\Omega$ in $\mathbb{R}^{N}$ in generalized divergence form as follows:

$$
A(u)=\sum_{|\beta| \leq \alpha}(-1)^{|\beta|} D^{\beta} A_{\beta}\left(u, \nabla u, \ldots, \nabla^{\alpha} u\right) .
$$

The function $g$ satisfies a sign condition but has otherwise completely unrestricted growth with respect to $u$.

Equations of type (1) were first considered by Browder [1] as an application to the theory of not everywhere defined mapping of monotone type. For $\alpha=1$, that is, $A$ of second order, their solvability under fairly general and natural assumptions was proved by Hess [2]. The treatment of the case $\alpha>1$ is more involved due to the lack of a simple truncation operator in higher order Sobolev spaces. Webb [3] observed that rather delicate approximation procedure introduced in nonlinear potential theory by Hedberg [4] could be used in place of truncation. This yielded the solvability of (1) for $\alpha>1$. Brezis and Browder [5] then used this approximation procedure to solve a question which they had considered earlier [6] about the action of some distribution. They also showed that their result on the action of some distributions could itself be used in place of truncation in the study the problem (1). In a more general case, Boccardo et al. studied inequations associated with (1), see [7].

The functional setting in all the results mentioned above is that of the usual Sobolev spaces $W^{\alpha, p}\left(\mathbb{R}^{N}\right)$, and the functions $A_{\beta}$ in (2) are supposed to satisfy polynomial growth conditions with respect to $u$ and its derivatives. Benkirane and Gossez established this result in the Orlicz-Sobolev spaces $W^{\alpha} L_{A}\left(\mathbb{R}^{N}\right)$, see $[8-10]$.

It is our purpose in this paper to study these problems in this setting of Sobolev spaces with variable exponent $W^{\alpha, p(\cdot)}\left(\mathbb{R}^{N}\right)$ of the harder higher order case $\alpha>1$. We consider problem (1) as well as Hedberg's approximation theorem and Brezis-Browder's question on the action of some distributions.

The paper is structured as follows. After some necessary preliminaries, in Section 3, we give the proof of the approximation theorem. In addition, Section 4 forms a useful supplement to some applications of (1). 


\section{Preliminaries}

In this section we list briefly some definitions and well known facts about Sobolev spaces with variable exponent and Bessel potential spaces with variable exponent. Standard references are $[11,12]$.

Let $\Omega$ be an open subset of $\mathbb{R}^{N}$, by the symbol $\mathscr{P}(\Omega)$, we denote the family of all measurable functions $p(\cdot): \Omega \rightarrow$ $[1, \infty]$.

For $p(\cdot) \in \mathscr{P}(\Omega)$, put

$$
p^{+}=\operatorname{ess} \sup _{x \in \Omega} p(x), \quad p^{-}=\operatorname{ess} \inf _{x \in \Omega} p(x) .
$$

Furthermore, we introduce a class $\mathscr{B}(\Omega)$ by

$$
\mathscr{B}(\Omega):=\left\{p \in \mathscr{P}(\Omega) ; 1<p^{-} \leq p^{+}<\infty\right\} .
$$

Let $p(\cdot) \in \mathscr{B}(\Omega)$, and consider the functional

$$
\varrho_{p}(\cdot)(f)=\int_{\Omega}|f(x)|^{p(x)} d x,
$$

on all measurable function $f$ on $\Omega$. The Lebesgue space with variable exponent $L^{p(\cdot)}(\Omega)$ is defined as the set of all measurable functions $f$ on $\Omega$ such that, for some $\lambda>0$,

$$
\varrho_{p}(\cdot)\left(\frac{f}{\lambda}\right)<\infty
$$

equipped with the norm

$$
\|f\|_{p}(\cdot)=\inf \left\{\lambda>0 ; \varrho_{p(\cdot)}\left(\frac{f}{\lambda}\right) \leq 1\right\} .
$$

The space $\left(L^{p(\cdot)}(\Omega),\|\cdot\|_{p(\cdot)}\right)$ is a separable Banach space. Moreover, if $1<p_{-} \leq p_{+}<+\infty$, then $L^{p(\cdot)}(\Omega)$ is uniformly convex, hence, reflexive, and its dual space is isomorphic to $L^{p^{\prime}(\cdot)}(\Omega)$, where $1 / p(x)+1 / p^{\prime}(x)=1$.

Finally, we have the Holder type inequality as follows:

$$
\left|\int_{\Omega} u v d x\right| \leq\left(\frac{1}{p_{-}}+\frac{1}{p_{-}^{\prime}}\right)\|u\|_{p(\cdot)}\|v\|_{p^{\prime}(\cdot)}
$$

for all $u \in L^{p(\cdot)}(\Omega)$ and $v \in L^{p^{\prime}(\cdot)}(\Omega)$.

The Hardy-Little-wood maximal operator $M$ is defined on locally integrable functions $f$ on $\Omega$ by the following formula:

$$
M f(x)=\sup _{R>0} \frac{1}{|B(x, R)|} \int_{B(x, R)}|f(y)| d y,
$$

where $B(x, R)$ denote the open ball in $\Omega$ with center $x$ and radius $R$, and $|B(x, R)|$ denotes the volume of $B(x, R)$.

Definition 1. By $\mathscr{M}(\Omega)$, denote the class of all functions $p \in$ $\mathscr{B}(\Omega)$ for which the operator $M$ is bounded on $L^{p(\cdot)}(\Omega)$; that is,

$$
\|M f\|_{p}(\cdot) \leq C\|f\|_{p}(\cdot)
$$

with a positive constant $C$ independent of $f$.
Remark 2. For example, $p(\cdot) \in \mathscr{M}(\Omega)$ if the following two conditions are satisfied:

$$
\begin{aligned}
& |p(x)-p(y)| \leq \frac{c}{-\log (|x-y|)}, \quad|x-y| \leq \frac{1}{2}, \\
& |p(x)-p(y)| \leq \frac{c}{\log (e+|x|)}, \quad|y|>|x| .
\end{aligned}
$$

For more details, see [13-16], where various sufficient conditions for $p(\cdot) \in \mathscr{M}(\Omega)$ can be found.

Let $p(\cdot) \in \mathscr{B}(\Omega)$ and $\alpha \in \mathbb{N}$; we define the Sobolev space with variable exponent by

$$
W^{\alpha, p(\cdot)}(\Omega)=\left\{u, D^{\beta} u \in L^{p(\cdot)}(\Omega) \text { if }|\beta| \leq \alpha\right\},
$$

equipped with the norm

$$
\|u\|_{W^{\alpha, p(\cdot)}}=\sum_{|\beta| \leq \alpha}\left\|D^{\beta} u\right\|_{L^{p(\cdot)}},
$$

where $\beta \in \mathbb{N}_{0}^{N}$ is a multi-index, $|\beta|=\beta_{1}+\cdots+\beta_{N}$, and $D^{\beta}=\partial^{|\beta|} / \partial^{\beta_{1}} x_{1} \cdots \partial^{\beta_{N}} x_{N}$.

Next, we define $W_{0}^{\alpha, p(\cdot)}(\Omega)$ as the closure of $\mathscr{D}(\Omega)$ in $W^{\alpha, p(\cdot)}(\Omega)$ and $W^{-\alpha, p^{\prime}(\cdot)}(\Omega)$ the dual space of $W^{\alpha, p(\cdot)}(\Omega)$, where $1 / p(\cdot)+1 / p^{\prime}(\cdot)=1$. by

The Bessel kernel $G_{\alpha}$ (see [17]) of order $\alpha>0$ is defined

$$
\begin{array}{r}
G_{\alpha}(x)=\frac{\pi^{N / 2}}{\Gamma(\alpha / 2)} \int_{0}^{\infty} e^{-s-\pi^{2}|x|^{2} / s} s^{(\alpha-N) / 2} \frac{d s}{s}, \\
x \in \mathbb{R}^{N} .
\end{array}
$$

The Riesz kernel $I_{\alpha}$ (see [18]) of order $\alpha>0$ is defined by

$$
I_{\alpha}(x)=\frac{\gamma_{\alpha}}{|x|^{N-\alpha}}
$$

where $\gamma_{\alpha}$ is a certain constant, whose exact value is

$$
\gamma_{\alpha}=\Gamma \frac{((N-\alpha) / 2)}{\left(\pi^{N / 2} 2^{\alpha} \Gamma(\alpha / 2)\right)} .
$$

It follows easily that

$$
G_{\alpha}(x) \sim I_{\alpha}(x), \quad|x| \longrightarrow 0,0<\alpha<N,
$$

and an examination of (14) shows without much effort that for any $c<1$,

$$
\begin{aligned}
& G_{\alpha}(x)=o\left(e^{-c|x|}\right), \quad|x| \longrightarrow \infty, N>\alpha>0, \\
& \int_{|x-y|<\delta} \frac{f(y) d y}{|x-y|^{N-\alpha}} \leq A \delta^{\alpha} M f(x) .
\end{aligned}
$$

Writing $G_{\alpha}(x)=G_{\alpha}(r), r=|x|$, this implies that, for $N>\alpha>$ 1 ,

$$
\begin{gathered}
G_{\alpha}^{\prime}(r) \sim-(N-\alpha) G_{\alpha-1}(r), \quad r \longrightarrow 0, N>\alpha>0, \\
G_{\alpha}^{\prime}(r) \sim-a_{\alpha} r^{(\alpha-N-1) / 2} e^{-r} \sim-c G_{\alpha}(r), \quad r \longrightarrow \infty,
\end{gathered}
$$

with $c=2^{(\alpha+N-3) / 2} \pi^{(N-\alpha-1) / 2}$ and $1 / a_{\alpha}=(4 \pi)^{(\alpha) / 2} \Gamma((\alpha) / 2)$. 


\section{Main Results}

First, we give the following results which will be used in our main result.

3.1. Useful Results. Let $p(\cdot) \in \mathscr{B}\left(\mathbb{R}^{N}\right)$ and $\alpha>0$. The Bessel potential space with variable exponent $L^{\alpha, p(\cdot)}\left(\mathbb{R}^{N}\right)$ is defined, for $N>\alpha>0$, by

$$
L^{\alpha, p(\cdot)}\left(\mathbb{R}^{N}\right)=\left\{u=G_{\alpha} * f ; f \in L^{p(\cdot)}\left(\mathbb{R}^{N}\right)\right\},
$$

and is equipped with the norm

$$
\|u\|_{\alpha, p(\cdot)}=\|f\|_{p(\cdot)} .
$$

Lemma 3 (see [12]). If $p(\cdot) \in \mathscr{M}\left(\mathbb{R}^{N}\right)$ and $\alpha \in \mathbb{N}$, then

$$
L^{\alpha, p(\cdot)}\left(\mathbb{R}^{N}\right) \cong W^{\alpha, p(\cdot)}\left(\mathbb{R}^{N}\right)
$$

and the corresponding norms are equivalent.

Lemma 4 (see [12]). Suppose that $p(\cdot) \in \mathscr{M}\left(\mathbb{R}^{N}\right)$ and $\alpha \geq 0$. Then there exists a positive constant $C$ such that

$$
\left\|G_{\alpha} * f\right\|_{p(\cdot)} \leq C\|f\|_{p(\cdot)}, \quad \text { for } f \in L^{p(\cdot)}\left(\mathbb{R}^{N}\right) .
$$

We will now verify that $u_{n}$ satisfies all the required properties in Proposition 7. The argument relies heavily on the following Lemmas 5 and 6 . In the sequel, we need the following two technical lemmas.

Lemma 5. If $1<p^{-} \leq p(x) \leq p^{+}<\infty$ and for any multiindex $|\xi|<\alpha<N$, there exists a constant $A$ such that for any $f \in L^{p(\cdot)}\left(\mathbb{R}^{N}\right)$,

$$
\begin{array}{r}
\left|D^{\xi}\left(G_{\alpha} * f(x)\right)\right| \leq A(M f(x))^{|\xi| / \alpha}\left(G_{\alpha} *|f|(x)\right)^{1-|\xi| / \alpha}, \\
\text { for almost every } x \in \mathbb{R}^{N} .
\end{array}
$$

Proof. We assume that $M f(x)<\infty$; otherwise, there is nothing to prove. We then observe that there is a constant $A_{1}$ such that $G_{\alpha} *|f|(x) \leq A_{1} M f(x)$. In fact, by (17), (18), and ([11], Lemma 6.1.4), there exists a constant $A$ such that

$$
\begin{aligned}
G_{\alpha} & *|f|(x) \\
\leq & A \int_{|x-y|<1} \frac{|f(y)| d y}{|x-y|^{N-\alpha}} \\
& +A \int_{|x-y| \geq 1}|f(y)| e^{-|x-y| / 2} d y \\
\leq & A M f(x)+A \sum_{i=1}^{\infty} e^{-i / 2} \int_{i+1>|x-y| \geq i}|f(y)| d y \\
\leq & A M f(x)+A M f(x) \sum_{i=1}^{\infty}(i+1)^{N} e^{-i / 2}=A_{1} M f(x) .
\end{aligned}
$$

Then, by (19) and (18), for any $\delta \leq 1$, we have

$$
\begin{aligned}
& \left|D^{\xi}\left(G_{\alpha} *|f|(x)\right)\right| \\
& \leq A \int_{|x-y|<\delta} \frac{|f(y)| d y}{|x-y|^{N-\alpha+|\xi|}} \\
& \quad+A \int_{\delta \leq|x-y|<1} \frac{|f(y)| d y}{|x-y|^{N-\alpha+|\xi|}} \\
& \quad+A \int_{|x-y| \geq 1} G_{\alpha}(x-y)|f(y)| d y \\
& \leq A\left(\delta^{\alpha-|\xi|} M f(x)+\delta^{-|\xi|}\left(G_{\alpha} *|f|(x)\right)\right. \\
& \left.\quad+\left(G_{\alpha} *|f|(x)\right)\right) .
\end{aligned}
$$

Now choosing $\delta^{\alpha}=G_{\alpha} *|f|(x) / A_{1} M f(x)$; then, $\delta \leq 1$ and the result follows.

Lemma 6. Suppose that $1<p^{-} \leq p(x) \leq p^{+}<\infty$ and $0<\alpha<N$. Let $H \in C^{k}\left(\mathbb{R}^{+}\right)$for some $k \geq \alpha$, if $H$ satisfies

$$
\sup _{t>0}\left|t^{i-1} H^{(i-1)}(t)\right| \leq L<\infty, \quad \text { with } i=1,2, \ldots, k .
$$

Then $H\left(G_{\alpha} * f\right) \in L^{\alpha, p(\cdot)}\left(\mathbb{R}^{N}\right)$ for every $f \in L_{+}^{p(\cdot)}\left(\mathbb{R}^{N}\right)$, and there exists a constant $A$, depending only on $\alpha$ and $N$, such that

$$
\left\|H\left(G_{\alpha} * f\right)\right\|_{\alpha, p(\cdot)} \leq A L\left\|G_{\alpha} * f\right\|_{\alpha, p(\cdot)}=A L\|f\|_{p(\cdot)} .
$$

Proof. For $\alpha$ integer. Assume that $f \in C_{0}^{\infty}\left(\mathbb{R}^{N}\right)$ and $f \geq 0$. Set $u=G_{\alpha} * f$ and notice that $u(x)>0$ for all $x$, so that $H(u)$ is defined. If $\xi$ is a multi-indix with $|\xi|=\alpha$, we find by the chain rule that

$$
D^{\xi}(H(u))=\sum_{i=1}^{\alpha} H^{(i)}(u) \sum c_{\xi} D^{\xi^{1}} u \cdots D^{\xi^{i}} u,
$$

where the interior sum is over all ordered $i$-tuples of multiindices $\left\{\xi^{1}, \ldots, \xi^{i}\right\}$ such that $\xi^{1}+\cdots+\xi^{i}=\xi$, and all $\left|\xi^{j}\right| \geq 1$. The $c_{\xi}$ are coefficients, whose exact value is of no consequence to us. Thus, by assumption of Lemma 3.4 we get

$$
\left|D^{\xi}(H(u))\right| \leq A L \sum_{i=1}^{\alpha} u^{1-i} \sum\left|D^{\xi^{1}} u \cdots D^{\xi^{i}} u\right| .
$$

For $i>1$, we estimate these derivatives by means of Lemma 5 . By the positivity of $f$, we have

$$
\left|D^{\xi^{j}} u\right| \leq A(M f(x))^{\left|\xi^{j}\right| / \alpha} u^{1-\left|\xi^{j}\right| / \alpha} .
$$

Thus, since $\sum_{j=1}^{i}\left(1-\left|\xi^{j}\right| / \alpha\right)=i-|\xi| / \alpha=i-1$,

$$
\sum_{i=2}^{\alpha} u^{1-i} \sum\left|D^{\xi^{1}} u \cdots D^{\xi^{i}} u\right| \leq A \sum_{i=2}^{\alpha} u^{1-i} M f u^{i-1}=A M f .
$$

Taking the term with $i=1$ into account, we obtain

$$
\left|D^{\xi}(H(u))\right| \leq A L\left(|M f|+\left|D^{\xi} u\right|\right) .
$$


But we already know from (10) that $\|M f\|_{p(\cdot)} \leq A\|f\|_{p(\cdot)}$ and that $\left\|D^{\xi}\left(G_{\alpha} * f\right)\right\|_{p(\cdot)} \leq A\|f\|_{p(\cdot)}$ for $|\xi|=\alpha$.

This finishes the proof for smooth $f$.

Now we pass to the general case and let $f$ be an arbitrary function in $L_{+}^{p(\cdot)}\left(\mathbb{R}^{N}\right)$. Then there are nonnegative functions $f_{i} \in C_{0}^{\infty}\left(\mathbb{R}^{N}\right), i=1,2, \ldots$, such that

$$
\lim _{i \rightarrow \infty}\left\|f_{i}-f\right\|_{p(\cdot)}=0 .
$$

By the first part of the proof,

$$
\left\|H\left(\left(G_{\alpha} * f_{i}\right)\right)\right\|_{\alpha, p(\cdot)} \leq A L\|f\|_{p(\cdot)},
$$

for all sufficiently large $i$.

Thus, setting $H\left(\left(G_{\alpha} * f_{i}\right)\right)=G_{\alpha} * g_{i}$, we can assume that $\left\{g_{i}\right\}_{1}^{\infty}$ converges weakly in $L^{p(\cdot)}\left(\mathbb{R}^{N}\right)$ to an element $g$, with $\|g\|_{p(\cdot)} \leq A L\|f\|_{p(\cdot)}$.

We have to prove that $G_{\alpha} * g=H\left(\left(G_{\alpha} * f\right)\right)$.

The strong convergence of $\left\{f_{i}\right\}_{1}^{\infty}$ and the fact that $G_{\alpha} \in$ $L^{1}\left(\mathbb{R}^{N}\right)$ imply, by Lemma 4 , that $\left\{G_{\alpha} * f_{i}\right\}_{1}^{\infty}$ converges strongly in $L^{p(\cdot)}\left(\mathbb{R}^{N}\right)$ to $G_{\alpha} * f$. After extraction of a subsequence, we can assume that

$$
\lim _{i \rightarrow \infty} G_{\alpha} * f_{i}(x)=G_{\alpha} * f(x) \quad \text { a.e. }
$$

But $H$ is continuous, so it follows that

$$
\begin{aligned}
\lim _{i \rightarrow \infty} G_{\alpha} * g_{i}(x) & =\lim _{i \rightarrow \infty} H\left(\left(G_{\alpha} * f_{i}\right)\right)(x) \\
& =H\left(\left(G_{\alpha} * f\right)\right)(x) \text { a.e. }
\end{aligned}
$$

On the other hand, the weak convergence of $\left\{g_{i}\right\}_{1}^{\infty}$ implies that the pointwise limit of $\left\{G_{\alpha} * g_{i}\right\}_{1}^{\infty}$ (which is now known to exist a.e.) is $G_{\alpha} * g$. In fact, setting $g_{i}-g=h_{i}$, for an arbitrary $\epsilon>0$,

$$
\begin{aligned}
G_{\alpha} * h_{i}(x)= & \int_{|x-y| \leq \epsilon} G_{\alpha}(x-y) h_{i}(y) d y \\
& +\int_{|x-y|>\epsilon} G_{\alpha}(x-y) h_{i}(y) d y .
\end{aligned}
$$

By weak convergence, the last term tends to zero, since $G_{\alpha}$ is in $L^{p^{\prime}(\cdot)}\left(\mathbb{R}^{N}\right)$ away from the origin. Consider the following:

$$
\text { Let } \lambda>\liminf _{i \rightarrow \infty}\left\|\int_{|y| \leq \epsilon} G_{\alpha}(y) h_{i}(\cdot-y) d y\right\|_{p(\cdot)} \text {. }
$$

We deduce that, for all $j, \lambda>$ $\inf _{i \geq j}\left\|\int_{|y| \leq \epsilon} G_{\alpha}(y) h_{i}(\cdot-y) d y\right\|_{p(\cdot)}$, which implies that for all $j$, there exists $i \geq j$, such that

$$
\lambda>\left\|\int_{|y| \leq \epsilon} G_{\alpha}(y) h_{i}(\cdot-y) d y\right\|_{p(\cdot)} .
$$

Then,

$$
\begin{aligned}
& \int_{|y| \leq \epsilon}\left(\frac{\liminf _{i \rightarrow \infty} G_{\alpha}(y) h_{i}(x-y)}{\lambda}\right)^{p(x)} d x \\
& \quad \leq \liminf _{i \rightarrow \infty} \int_{|y| \leq \epsilon}\left(\frac{G_{\alpha}(y) h_{i}(\cdot-y)}{\lambda}\right)^{p(x)} d x \leq 1 .
\end{aligned}
$$

Since $\lambda \geq\left\|\lim _{i n f}{ }_{i \rightarrow \infty} G_{\alpha} * h_{i}\right\|_{p(\cdot)}$, then we have

$$
\begin{aligned}
\left\|\lim _{i \rightarrow \infty} G_{\alpha} * h_{i}\right\|_{p(\cdot)} & \leq \liminf _{i \rightarrow \infty}\left\|\int_{|y| \leq \epsilon} G_{\alpha}(y) h_{i}(\cdot-y) d y\right\|_{p(\cdot)} \\
& \leq \sup _{i}\left\|h_{i}\right\|_{p(\cdot)} \int_{|y| \leq \epsilon} G_{\alpha}(y) d y,
\end{aligned}
$$

which is an arbitrary small number, and thus for a.e. $x$,

$$
\begin{aligned}
G_{\alpha} * g(x) & =\lim _{i \rightarrow \infty} G_{\alpha} * g_{i}(x) \\
& =\lim _{i \rightarrow \infty} H\left(\left(G_{\alpha} * f_{i}\right)\right)(x) \\
& =H\left(\left(G_{\alpha} * f\right)\right)(x) .
\end{aligned}
$$

This completes the proof of Lemma 5.

Proposition 7. Let $u \in W^{\alpha, p(\cdot)}\left(\mathbb{R}^{N}\right)$, there exist a sequence $u_{n}$, such that

(i) $u_{n} \in W^{\alpha, p(\cdot)}\left(\mathbb{R}^{N}\right) \cap L^{\infty}\left(\mathbb{R}^{N}\right)$, supp $u_{n}$ is compact;

(ii) $\left|u_{n}(x)\right| \leq|u(x)|$ and $u_{n}(x) u(x) \geq 0$ a.e. in $\mathbb{R}^{N}$;

(iii) $u_{n} \rightarrow u$ in $W^{\alpha, p(\cdot)}\left(\mathbb{R}^{N}\right)$ as $n \rightarrow \infty$.

Proof. The proof of Proposition 7 is done in two steps as follows.

Step 1 (case $\left.\alpha p^{-}>N\right)$. Let $\xi \in C_{0}^{\infty}\left(\mathbb{R}^{N}\right)$ be a fixed function such that $0 \leq \xi \leq 1$ and $\xi(x)=1$ in a neighborhood of the origin.

Let $\xi_{n}(x)=\xi(x / n)$; then, $u_{n}(x)=\xi_{n}(x) u(x)$ satisfies all the required properties, using the fact that $u \in L^{\infty}(\Omega)$ by Sobolev's theorem [19].

Step 2 (case $\left.\alpha p^{-} \leq N\right)$. We assume that $u$ has compact support, if necessary by multiplying with a suitable $\xi_{n}$. We represent $u$ as a Bessel potential, $u=G_{\alpha} * f$, so that $\|f\|_{p(\cdot)} \leq$ $A\|u\|_{W^{\alpha ; p(\cdot)} .}$ Set

$$
v=G_{\alpha} *|f|,
$$

and let $T \in C^{\infty}(\mathbb{R})$ be a function such that $0 \leq T \leq 1, T(t)=1$ for $0 \leq t \leq 1 / 2$ and $T(t)=0$ for $t \geq 1$. Then, set

$$
u_{n}(x)=T\left(\frac{v(x)}{n}\right) u(x), \quad n=1,2,3, \ldots
$$

We First observe that, $u_{n}(x)=0$ on the set $\{x: v(x) \geq n\}$, which includes $\{x:|u(x)| \geq n\}$, and so we have $\left|u_{n}(x)\right|<n$ a.e., and thus $u_{n}(x) u(x) \geq 0$. It remains to prove that $u_{n} \in$ $W^{\alpha, p(\cdot)}\left(\mathbb{R}^{N}\right)$ and that $u_{n}$ converges to $u$ as $n$ tends to $\infty$.

Let $\eta$ be any multi-index with $0<|\eta|=\beta \leq \alpha$. If $\eta=$ $\eta^{1}+\cdots+\eta^{i}, i>1$, and all $\left|\eta^{j}\right| \geq 1$, we find by the same arguments as in the proof of Lemma 6 that

$$
\left|D^{\eta} T\left(\frac{v(x)}{n}\right)\right| \leq A \sum_{i=1}^{\beta} n^{-i} \sum\left|D^{\eta^{1}} v(x) \cdots D^{\eta^{i}} v(x)\right| .
$$


By Lemma 5, we have, for any multi-index $\eta$ with $0<|\eta|<\alpha$,

$$
\left|D^{\eta} v(x)\right| \leq A M f(x)^{|\eta| / \alpha}(v(x))^{1-|\eta| / \alpha} .
$$

On the open set $\{x: v(x)>n\}$, using the fact that $D^{\eta} T(v(x) / n)=0$; then,

$$
\begin{aligned}
\left|D^{\eta} H\left(\frac{v(x)}{n}\right)\right| & \leq A \sum_{i=1}^{\beta} n^{-i} n^{i-|\eta| / \alpha} M f(x)^{|\eta| / \alpha} \\
& \leq A n^{-|\eta| / \alpha} M f(x)^{|\eta| / \alpha}
\end{aligned}
$$

for $|\eta| \leq \alpha-1$, and

$$
\left|D^{\eta} T\left(\frac{v(x)}{n}\right)\right| \leq A n^{-1}\left(M f(x)+\left|D^{\eta} v(x)\right|\right),
$$

for $|\eta|=\alpha$.

By using Leibniz's formula, we have for $|\xi|=\alpha$, if $v(x) \leq n$, again using Lemma 5, that

$$
\begin{aligned}
& \left|D^{\xi} u_{n}(x)-D^{\xi} u(x)\right|=\left|\left(1-T\left(\frac{v(x)}{n}\right)\right) D^{\xi} u(x)\right| \\
& \left|D^{\xi} u_{n}(x)-D^{\xi} u(x)\right| \\
& \leq A \sum_{0<|\eta|<\alpha}\left|D^{\eta} T\left(\frac{v(x)}{n}\right)\right|\left|D^{\xi-\eta} u(x)\right| \\
& \quad+\left|D^{\xi} H\left(\frac{v(x)}{n}\right)\right||u(x)| \\
& \leq A \sum_{0<\beta<\alpha} n^{-\beta / \alpha} M f(x)^{\beta / \alpha} M f(x)^{1-\beta / \alpha} v(x)^{\beta / \alpha} \\
& \quad+A n^{-1}\left(M f(x)+\left|D^{\xi} v(x)\right|\right) v(x) \\
& \leq A\left(\frac{v(x)}{n}\right)^{1 / \alpha} M f(x) \\
& \quad+A\left(\frac{v(x)}{n}\right)\left(M f(x)+\left|D^{\xi} v(x)\right|\right) .
\end{aligned}
$$

If $v(x)>n$, we have $D^{\xi} u_{n}(x)=0$. It follows that a.e.

$$
\begin{gathered}
\lim _{n \rightarrow \infty}\left|D^{\xi} u_{n}(x)-D^{\xi} u(x)\right|=0, \\
\left|D^{\xi} u_{n}(x)-D^{\xi} u(x)\right| \leq\left|D^{\xi} u(x)\right|+A\left(M f(x)+\left|D^{\xi} v(x)\right|\right) .
\end{gathered}
$$

The functions on the right hand side belong to $L^{p(\cdot)}$, so the theorem follows by applying the dominated convergence.

Remark 8 . The sequence $u_{n}$ constructed above satisfies

$$
\left\|u_{n}\right\|_{W^{\alpha, p(\cdot)}} \leq C\|u\|_{W^{\alpha, p(\cdot)}},
$$

with a constant $C$ depending only on $\alpha$ and $N$.
3.2. Existence Result. This subsection is devoted to establish the following existence theorem.

Theorem 9. Let $T \in L_{\text {loc }}^{1}\left(\mathbb{R}^{N}\right) \cap W^{-\alpha, p^{\prime}(\cdot)}\left(\mathbb{R}^{N}\right)$ and $u \in$ $W^{\alpha, p(\cdot)}\left(\mathbb{R}^{N}\right)$. Assume that $T(x) u(x) \geq h(x)$ a.e. in $\mathbb{R}^{N}$, for some $h \in L^{1}\left(\mathbb{R}^{N}\right)$. Then,

$$
T u \in L^{1}\left(\mathbb{R}^{N}\right), \quad \int_{\mathbb{R}^{N}} T(x) u(x) d x=\langle T, u\rangle .
$$

Proof. We first deduce Theorem 9 as a simple consequence of Proposition 7. Let $u_{n}$ be a sequence defined in Proposition 7. It follows easily from (i) in Proposition 7 (using convolution with mollifiers and according to [11]) that

$$
\int_{\mathbb{R}^{N}} T(x) u_{n}(x) d x=\left\langle T, u_{n}\right\rangle .
$$

By Proposition 7, the right hand side of (54) converges as $n \rightarrow \infty$ to $\langle T, u\rangle$. On the other hand, we have $T u_{n} \geq$ $-|h|$ a.e. We deduce from Fatou's lemma that $T u \in L^{1}$. We conclude by dominated convergence that

$$
\begin{gathered}
\int T(x) u_{n}(x) d x \rightarrow \int T(x) u(x) d x \\
\text { and thus } \int T(x) u(x) d x=\langle T, u\rangle .
\end{gathered}
$$

Theorem 10. Let $T \in L_{l o c}^{1}(\Omega) \cap W^{-\alpha, p^{\prime}(\cdot)}(\Omega)$ be such that

$$
\int_{\Omega \cap B(0, R)}|T(x)| d x<\infty \quad \text { for every } R<\infty .
$$

Assume that $u \in W_{0}^{\alpha, p(\cdot)}(\Omega)$ and $T(x) u(x) \geq h(x)$ a.e. in $\Omega$, for some $h \in L^{1}(\Omega)$. Then,

$$
T u \in L^{1}(\Omega), \quad \int_{\Omega} T(x) u(x) d x=\langle T, u\rangle .
$$

Proof. The proof is straightforward when $\alpha p^{-}>N$; therefore, we may assume that $\alpha p^{-} \leq N$. Using $\xi_{n} u$ in place of $u$, we may always reduce to the case where supp $u$ is bounded. Set

$$
\bar{u}= \begin{cases}u(x) & \text { if } x \in \Omega, \\ 0 & \text { if } x \in \mathbb{R}^{N} \backslash \Omega .\end{cases}
$$

Then $\bar{u} \in W^{\alpha, p(\cdot)}\left(\mathbb{R}^{N}\right)$. This allows us to write

$$
\bar{u}=G_{\alpha} * f,
$$

for some $f$ in $L^{p(\cdot)}\left(\mathbb{R}^{N}\right)$. As in the proof of Proposition 7, set

$$
v=G_{\alpha} *|f|, \quad u_{n}=H\left(\frac{v}{n}\right) u .
$$

Since $u \in W_{0}^{\alpha, p(\cdot)}(\Omega)$, there exists a sequence $u^{j} \in C_{0}^{\infty}(\Omega)$ such that $u^{j} \rightarrow u$ in $W^{\alpha, p(\cdot)}(\Omega)$ and a.e. (see [19]). For each $j$, we perform the above construction and we set

$$
\begin{aligned}
& \bar{u}^{j}=G_{\alpha} * f^{j}, \\
& v^{j}=G_{\alpha} *\left|f^{j}\right|, \quad u_{n}^{j}=H\left(\frac{v^{j}}{n}\right) u^{j} .
\end{aligned}
$$


Fix $\xi \in C_{0}^{\infty}\left(\mathbb{R}^{N}\right)$. We clearly have

$$
\int_{\Omega} T \xi u_{n}^{j} d x=\left\langle T, \xi u_{n}^{j}\right\rangle .
$$

As we keep $n$ fixed and let $j \rightarrow \infty$, we see that

$$
\int_{\Omega} T \xi u_{n}^{j} d x \longrightarrow \int_{\Omega} T \xi u_{n} d x
$$

by dominated convergence and (56).

On the other hand, by Remark 8, we obtain

$$
\left\|u_{n}^{j}\right\|_{W^{\alpha, p(\cdot)}(\Omega)} \leq\left\|\bar{u}^{j}\right\|_{W^{\alpha, p(\cdot)}\left(\mathbb{R}^{N}\right)} \leq C,
$$

where $C$ does not depend on $j$ and $n$.

Therefore, $u_{n}^{j}$ converges weakly to $u_{n}$ in $W_{0}^{\alpha, p(\cdot)}(\Omega)$ as $j \rightarrow$ $\infty$ and thus $\xi u_{n}^{j} \rightarrow \xi u_{n}$ as $j \rightarrow \infty$. Passing to the limit in (62) as $j \rightarrow \infty$, we find

$$
\int_{\Omega} T \xi u_{n}=\left\langle T, \xi u_{n}\right\rangle
$$

We conclude easily (by the argument as in the proof of Theorem 9) that

$$
T u \in L^{1}(\Omega), \quad \int_{\Omega} T(x) u(x) d x=\langle T, u\rangle .
$$

\section{An Application to a Strongly Nonlinear Elliptic Equation}

Let $\Omega$ be an open set on $\mathbb{R}^{N}$, and assume that $A$ : $W_{0}^{\alpha, p(\cdot)}(\Omega) \rightarrow W^{-\alpha, p^{\prime}(\cdot)}(\Omega)$ is a pseudomonotone operator which maps bounded sets into bounded sets and which is coercive. And let $g(x, s): \Omega \times \mathbb{R} \rightarrow \mathbb{R}$ be a Carathéodory function satisfying the sign condition $(\Omega \times \mathbb{R})$ and for each $t>0$, there exists $h_{t} \in L^{1}(\Omega)$ as follows:

$$
\sup _{|s| \leq t}|g(x, s)| \leq h_{t}(x) \text {. }
$$

Theorem 11. For every $f \in W^{-\alpha, p^{\prime}(\cdot)}(\Omega)$, there exists $u \in$ $W_{0}^{\alpha, p(\cdot)}(\Omega)$ such that

$$
\begin{gathered}
g(x, u) \in L^{1}(\Omega), \quad g(x, u) u \in L^{1}(\Omega), \\
\langle A u, v\rangle+\int_{\Omega} g(x, u) v d x=\langle f, v\rangle, \\
\forall v \in W_{0}^{\alpha, p(\cdot)}(\Omega) \cap L^{\infty}(\Omega) .
\end{gathered}
$$

Furthermore, if $g$ is nondecreasing in $u$ and if $u_{1}$ and $u_{2}$ are two solutions corresponding to $f_{1}$ and $f_{2}$, respectively, then

$$
\begin{aligned}
& \left\langle A u_{1}-A u_{2}, u_{1}-u_{2}\right\rangle \\
& \quad+\int_{\Omega}\left[g\left(x, u_{1}\right)-g\left(x, u_{2}\right)\right]\left(u_{1}-u_{2}\right) d x \\
& =\left\langle f_{1}-f_{2}, u_{1}-u_{2}\right\rangle .
\end{aligned}
$$

Proof. Let

$$
g_{n}(x, s)= \begin{cases}\eta\left(\frac{x}{n}\right) g(x, s) & \text { if }|g(x, s)| \leq n \\ \eta\left(\frac{x}{n}\right) n \operatorname{sign}(g(x, s)) & \text { if }|g(x, s)|>n\end{cases}
$$

where $\eta \in \mathscr{D}\left(\mathbb{R}^{N}\right)$ with $0 \leq \eta \leq 1$ and $\eta(x)=1$ near $x=0$. It follows easily from the theory of pseudomonotone operators that there exists $u_{n} \in W_{0}^{\alpha, p(\cdot)}(\Omega)$ such that

$$
A u_{n}+g_{n}\left(x, u_{n}\right)=f .
$$

In addition,

$$
\left\|u_{n}\right\|_{W_{0}^{\alpha, p(\cdot)}(\Omega)} \leq C, \quad \int_{\Omega} g_{n}\left(x, u_{n}\right) u_{n} \leq C .
$$

Then we can assume that

$$
\begin{gathered}
u_{n} \rightarrow u \quad \text { weakly in } W_{0}^{\alpha, p(\cdot)}(\Omega) \text {, a.e in } \Omega, \\
A u_{n} \rightarrow \chi \quad \text { weakly in } W^{-\alpha, p^{\prime}(\cdot)}(\Omega) .
\end{gathered}
$$

Moreover,

$$
\left|g_{n}\left(x, u_{n}\right)\right| \leq \sup _{|t| \leq s}|g(x, t)|+\frac{1}{s} g_{n}\left(x, u_{n}\right) u_{n},
$$

which implies that, for any measurable subset $E \subset \Omega$,

$$
\int_{E}\left|g_{n}\left(x, u_{n}\right)\right| \leq \int_{E} h_{s}(t)+\frac{C}{s} .
$$

Then, thanks to Vitali's theorem, we deduce that

$$
g_{n}\left(x, u_{n}\right) \longrightarrow g(x, u) .
$$

By Fatou's lemma, it is easy to see that

$$
\limsup _{n}\left\langle A u_{n}, u_{n}\right\rangle \leq\langle f, u\rangle-\int_{\Omega} g(x, u) d x .
$$

Set $T=g(x, u)=f-\chi$, we have $T \in L^{1}(\Omega) \cap W^{-\alpha, p^{\prime}(\cdot)}(\Omega)$ and as a consequence of Theorem 10 , we conclude that

$$
\int_{\Omega} g(x, u) u d x=\langle f-\chi, u\rangle .
$$

Therefore,

$$
\limsup _{n}\left\langle A u_{n}, u_{n}\right\rangle \leq\langle\chi, u\rangle \quad \text { and consequently } A u=\chi .
$$

The conclusion follows readily. (68) is again a direct consequence of Theorem 11.

\section{Conflict of Interests}

The authors declare that there is no conflict of interests regarding the publication of this paper. 


\section{References}

[1] F. E. Browder, "Existence theory for boundary value problems for quasi linear elliptic systems with strongly nonlinear lower order terms," in Proceedings of the Symposia in Pure Mathematics, vol. 23, pp. 269-286, The American Mathematical Society, 1971.

[2] P. Hess, "A strongly nonlinear elliptic boundary value problem," Journal of Mathematical Analysis and Applications, vol. 43, no. 1, pp. 241-249, 1973.

[3] J. R. L. Webb, "Boundary value problems for strongly nonlinear elliptic equations," Journal of the London Mathematical Society, vol. 2, no. 21, pp. 123-132, 1980.

[4] L. I. Hedberg, "Two approximation problems in function spaces," Arkiv för Matematik, vol. 16, no. 1, pp. 51-81, 1978.

[5] H. Brezis and F. E. Browder, "Some properties of higher order Sobolev spaces," Journal of Mathematical Analysis and Applications, vol. 245, pp. 0021-7824, 1982.

[6] H. Brezis and F. E. Browder, "A property of sobolev spaces," Communications in Partial Differential Equations, vol. 4, pp. 1077-1083, 1979.

[7] L. Boccardo, D. Giachetti, and F. Murat, "A generalization of a theorem of H. Brezis and F. E. Browder and applications to some unilateral problems," Annales de l'Institut Henri Poincaré C, vol. 7, pp. 367-384, 1990.

[8] A. Benkirane, "Approximation de type hedberg dans les espaces $W^{m} \operatorname{Llog} L(\Omega)$ et applications," Annales de la Faculté des Sciences de Toulouse, vol. 11, pp. 67-78, 1990.

[9] A. Benkirane and G. P. Gossez, "An approximation theorem for higer order Orlicz-Sobolev spaces," Studia Mathematica, vol. 92, pp. 231-255, 1989.

[10] A. Benkirane, "A theorem of H. Brezis and F. E. Browder type in Orlicz spaces and application," Pitman Research Notes in Mathematics Series, vol. 343, pp. 10-16, 1996.

[11] L. Diening, P. Harjulehto, P. Hästö, and M. Ružička, Lebesgue and Sobolev Spaces with Variable Exponents, vol. 2017 of Lecture Notes in Mathematics, Springer, Berlin, Germany, 2011.

[12] P. Gurka, P. Harjulehto, and A. Nekvinda, "Bessel potential spaces with variable exponent," Mathematical Inequalities and Applications, vol. 10, no. 3, pp. 661-676, 2007.

[13] D. Cruz-Uribe, A. Fiorenza, and C. J. Neugebauer, "Corrections to the maximal operator on variable $L^{p}$ spaces," Annales Academioe Scientiarum Fennica, vol. 29, pp. 247-249, 2004.

[14] D. Cruz-Uribe, A. Fiorenza, and C. J. Neugebauer, "The maximal function on variable $L^{p}$ spaces," Annales Academiae Scientiarum Fennicae Mathematica, vol. 28, no. 1, pp. 223-238, 2003.

[15] L. Diening, "Maximal function on generalized lebesgue spaces $L^{p(\cdot), " ~ M a t h e m a t i c a l ~ I n e q u a l i t i e s ~ a n d ~ A p p l i c a t i o n s, ~ v o l . ~ 7, ~ n o . ~ 2, ~}$ pp. 245-253, 2004.

[16] A. Nekvinda, "Hardy-Little-Wood maximal operator on $L^{p(\cdot)}\left(\mathbb{R}^{N}\right)$," Mathematical Inequalities and Applications, vol. 7, no. 2, pp. 255-266, 2004.

[17] D. R. Adams and L. I. Hedberg, Function Spaces and Potential Theory, Springer, New York, NY, USA, 1996.

[18] E. Stein, Singular Integrals and Differentiability Properties of Functions, Princeton University Press, Princeton, NJ, USA, 1970.

[19] X. L. Fan and D. Zhao, "On the spaces $L^{p(x)}(\Omega)$ and $W^{m, p(x)}(\Omega)$," Journal of Mathematical Analysis and Applications, vol. 236, pp. 424-446, 2001. 


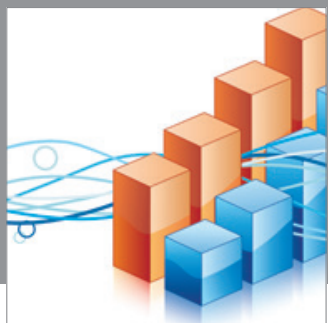

Advances in

Operations Research

mansans

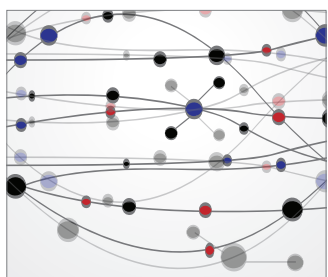

The Scientific World Journal
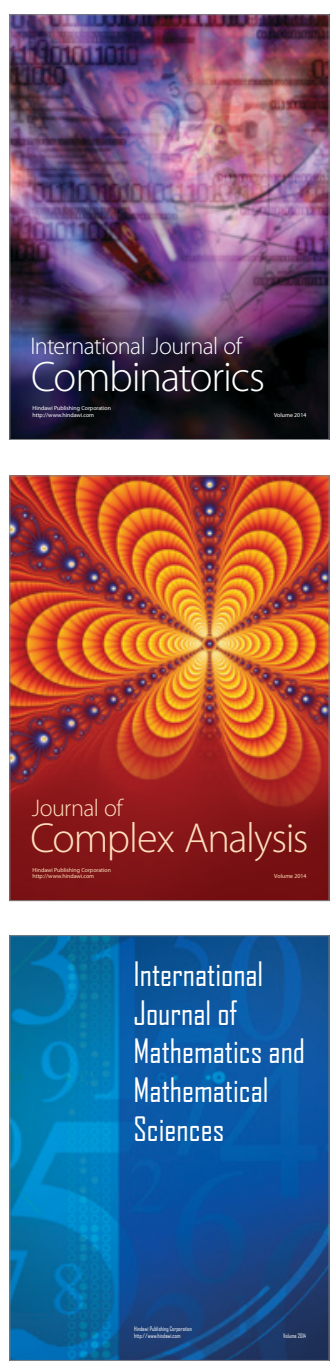
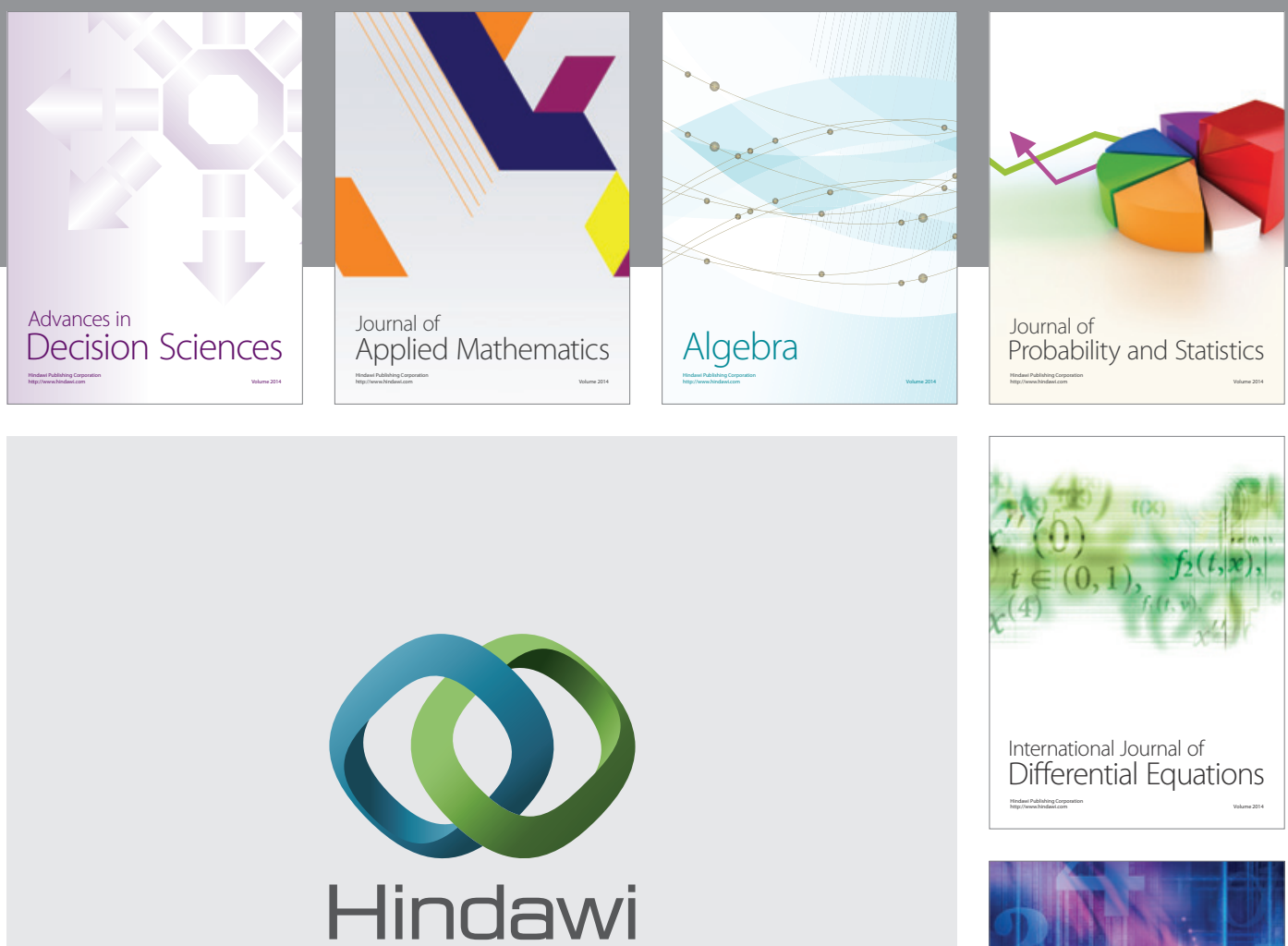

Submit your manuscripts at http://www.hindawi.com
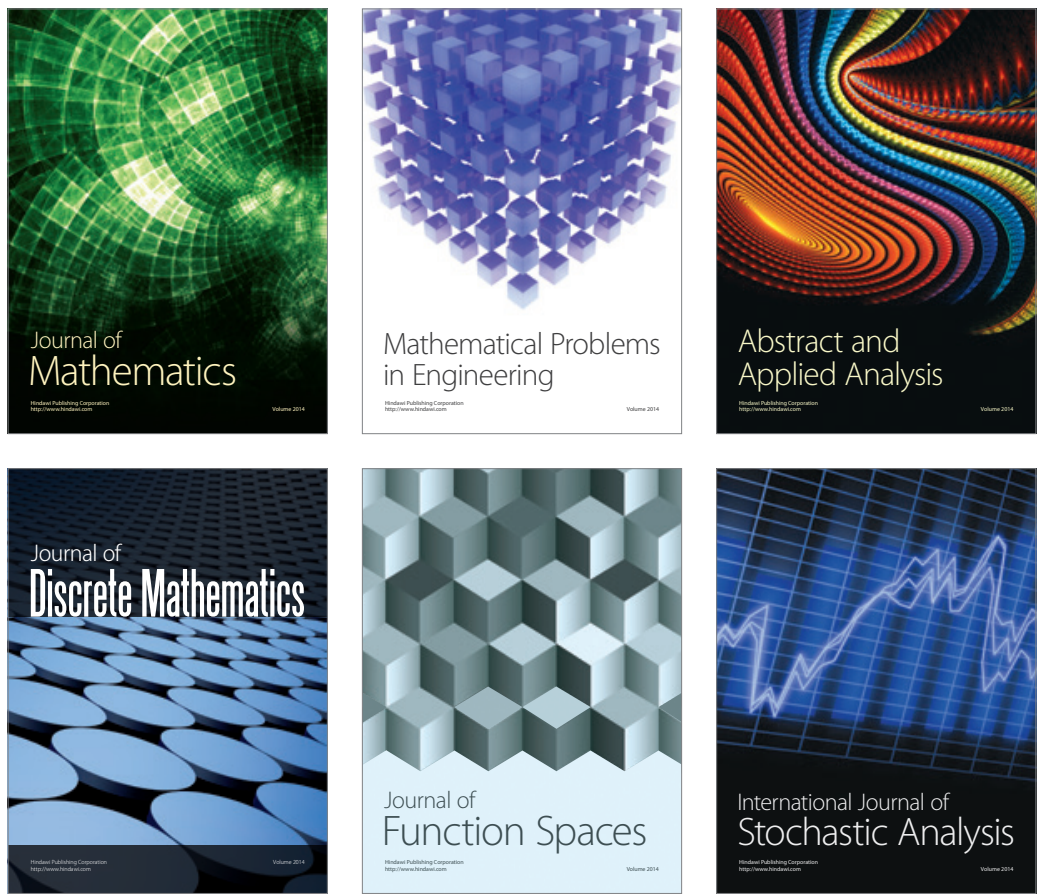

Journal of

Function Spaces

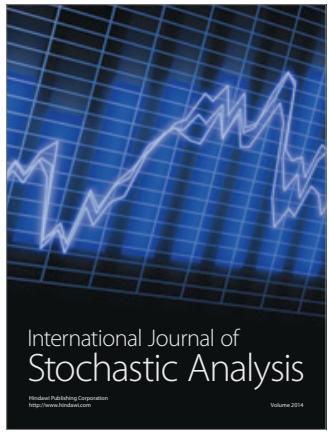

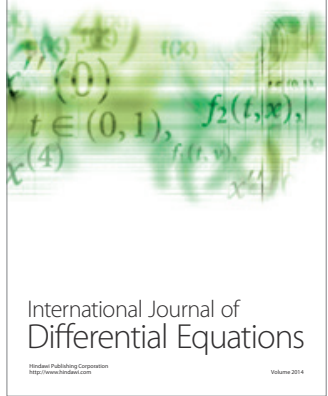
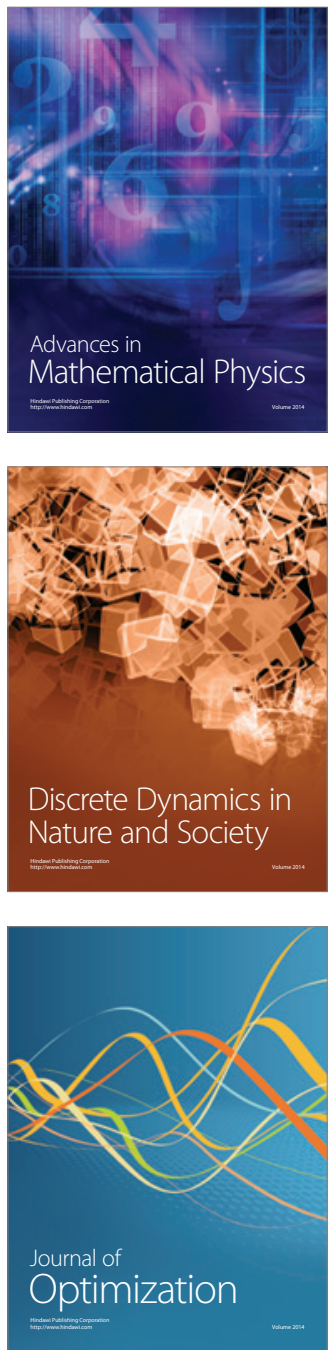